\title{
Second-Order Consensus in Multiagent Systems via Nonlinear Protocol
}

\author{
Huan Pan, ${ }^{1}$ Xiaohong Nian, ${ }^{2}$ and Ling Guo ${ }^{2}$ \\ ${ }^{1}$ School of Physics Electrical Information Engineering, Ningxia University, Yinchuan 750021, China \\ ${ }^{2}$ School of Information Science and Engineering, Central South University, Changsha 410075, China
}

Correspondence should be addressed to Huan Pan; pan198303@gmail.com

Received 8 February 2013; Accepted 15 April 2013

Academic Editor: Wenwu Yu

Copyright (C) 2013 Huan Pan et al. This is an open access article distributed under the Creative Commons Attribution License, which permits unrestricted use, distribution, and reproduction in any medium, provided the original work is properly cited.

This paper focuses on theoretical analysis of second-order consensus in multiagent system. As an extension of the general linear protocol, a nonlinear protocol is designed for multiagent system with undirected communication topology. The nonlinear protocol is also applied to achieve reference velocity consensus. Through choosing the appropriate Lyapunov functions and using LaSalle's invariance principle, some consensus conditions are derived. Simulation examples are provided to demonstrate the effectiveness of the proposed results.

\section{Introduction}

Recently, consensus problems have been investigated extensively in the content of distributed coordinated control of dynamic agents, partly owning to the potential broad application in various areas. A basic and fundamental question in consensus is how to design the effective protocols (or algorithms) such that each agent can achieve a common target. Most of the consensus literatures mainly considered general linear protocols for multiagent systems. However, the relation between agents is usually not linear. In some situations, the states of multiagent systems cannot be observed directly, and some nonlinear functions about the states can be observed. Therefore, it is necessary to design nonlinear protocols for multiagent systems.

Nonlinear functions are always used to describe the coupling relations of nodes in complex networks [1-6]. On the other hand, some scholars have paid attentions to nonlinear protocols for multiagent systems recently. Bauso et al. [7] designed the distributed nonlinear protocols and proposed a game theoretical approach to solve consensus problems. Xiao et al. [8] presented a new nonlinear protocol for state consensus of multiagent system which provides faster convergence rate than the typical linear protocol [9]. Liu et al. [10] discussed the consensus problem under two nonlinear protocols with directed topology. Hui and Haddad [11] developed a thermodynamic framework for addressing consensus for nonlinear multiagent dynamical systems. Shang [12] proposed some sufficient criteria guaranteeing multiagent systems to reach a consensus in finite time under nonlinear protocols. Li and Guan [13] investigated nonlinear consensus protocols for dynamic directed networks of multiagent systems based on the central manifold reduction technique. Shi and Hong [14] considered a group of continuoustime agents with nonlinear agent dynamics and concluded that the agents can flock to a convex target set. Sepulchre [15] introduced consensus problems whose underlying state space is not a linear space but a nonlinear space. Yu et al. [16] studied the consensus problem for cooperative agents with nonlinear dynamics in a directed network. Zhou and Wang [17] derived some sufficient conditions for (global/exponential) semistability for general discrete-time nonlinear protocols.

However, it is worthwhile to note that the abovementioned nonlinear protocols [7-13,17] are all proposed for the first-order multiagent systems. As we all know, firstorder consensus problems are mainly relative to communication topologies, but, for second-order consensus, both the interaction graph and the coupling strength affect the convergence result. Therefore, the consensus of second-order multiagent systems is more challenging and interesting and 
attracts researchers' broad attention. For example, Yu et al. [18] considered a second-order consensus problem for multiagent systems with nonlinear dynamics. Furthermore, Song et al. [19] studied the second-order leader-following consensus problem of nonlinear multiagent systems. Ren [20] proposed and analyzed a consensus algorithm for doubleintegrator dynamics with a bounded control input under an undirected interaction graph. Though [20] extended some existing results in consensus algorithms to account for actuator saturation, saturation function which is assumed to be the hyperbolic tangent function is concrete and simple. So far as we know, there is no result about designing some nonlinear protocols for second-order multiagent systems. For this purpose, this paper mainly designs a nonlinear protocol for second-order dynamics with an undirected communication topology as an extension of the linear protocol. Then, the consensus protocol for second-order multiagent system with a group reference velocity available to each individuality is proposed.

The rest of this paper is organized as follows. Section 2 provides some preliminary graph theory, the proposed nonlinear protocol, and two useful lemmas. In Section 3, consensus problem and reference velocity consensus of a secondorder multiagent system with an undirected communication topology are analyzed, respectively. Some numerical examples are given to show the effectiveness and advantage of the theoretical results in Section 4. Finally, a conclusion is provided in Section 5.

\section{Notation and Preliminaries}

2.1. Graph Theory. In this paper, the communication topology among $n$ multiagents is assumed to be an undirected graph $\mathscr{G}=(\mathscr{V}, \mathscr{E}, \mathscr{A})$, where $\mathscr{V}=\left\{v_{1}, \ldots, v_{n}\right\}$ is the set of nodes, $\mathscr{E} \subseteq \mathscr{V} \times \mathscr{V}$ is the set of edges, and $\mathscr{A}=$ $\left[a_{i j}\right] \in \mathbb{R}^{n \times n}$ represents the weighted adjacency matrix with nonnegative adjacency elements $a_{i j}$. The node or multiagent indexes belong to a finite index set $\mathscr{I}=\{1,2, \ldots, n\}$. An edge $e_{i j}=\left(v_{i}, v_{j}\right) \in \mathscr{E}$ in weighted undirected graph $\mathscr{G}$ denotes that node $j$ can receive information from $i$ each other. The adjacency elements associated with the edges of an undirected graph are positive $a_{i j}>0$ if $e_{i j} \in \mathscr{E}, a_{i j}=0$ otherwise, and have the property that $a_{i j}=a_{j i}$, for all $i \neq j$, since $e_{i j}$ implies $e_{j i}$. A path on $\mathscr{G}$ from node $i_{1}$ to $i_{l}$ is a sequence of undirected edges in the form of $\left(i_{k}, i_{k+1}\right), k=$ $1, \ldots, l-1$. An undirected graph is called connected if there exists a path from any node $i$ to any other node $j$.

2.2. Nonlinear Protocols for Second-Order Dynamics. Consider a second-order multiagent system with the following dynamics:

$$
\begin{aligned}
& \dot{x}_{i}(t)=v_{i}(t), \quad i \in \mathscr{I}, \\
& \dot{v}_{i}(t)=u_{i}(t),
\end{aligned}
$$

where $x_{i}(t) \in \mathbb{R}$ is the position state, $v_{i}(t) \in \mathbb{R}$ is the velocity state, and $u_{i}(t) \in \mathbb{R}$ is the control input (or protocol). Given the dynamical system (1), it is said that protocol $u_{i}(t)$ asymptotically solves the consensus problem, if the states of agents satisfy

$$
\begin{aligned}
& \lim _{t \rightarrow+\infty}\left|x_{i}(t)-x_{j}(t)\right| \\
& \quad=0, \lim _{t \rightarrow+\infty}\left|v_{i}(t)-v_{j}(t)\right|=0, \quad \forall i \neq j,
\end{aligned}
$$

for any $x_{i}(0)$ and $v_{i}(0)$.

The linear protocol $[21,22]$ is proposed as follows:

$$
u_{i}(t)=-\sum_{j=1}^{n} a_{i j}\left[\alpha\left(x_{i}(t)-x_{j}(t)\right)+\beta\left(v_{i}(t)-v_{j}(t)\right)\right]
$$

where $a_{i j}$ is the $(i, j)$ entry of the weighted adjacency matrix $\mathscr{A} \in \mathbb{R}^{n \times n}$, and parameters $\alpha, \beta$ are the coupling strengths.

It is clear that protocol (3) is based on a linear function of difference between each agent and other ones. Linear protocol (3) is simple and easy to use. However, sometimes in order to improve convergence rate or model a bounded control input, the linear protocol is often needed to be extended. Furthermore, in some cases, the state feedback $x_{i}(t)-x_{j}(t)$ may be unobservable; instead, we can observe a nonlinear function $\phi\left(x_{i}(t)-x_{j}(t)\right)$ of $x_{i}(t)-x_{j}(t)$. Naturally, a nonlinear protocol for second-order system (1) is designed as follows:

$$
\begin{aligned}
u_{i}(t)=-\sum_{j=1}^{n} a_{i j}( & \phi\left(x_{i}(t)-x_{j}(t)\right) \\
& \left.+\psi\left(v_{i}(t)\right)-\psi\left(v_{j}(t)\right)\right),
\end{aligned}
$$

where $a_{i j}$ is the $(i, j)$ entry of the weighted adjacency matrix $\mathscr{A}$ and $\phi(\cdot)$ and $\psi(\cdot)$ are two continuous functions.

In order to ensure existence, uniqueness of solution, and the operative property of nonlinear functions $\phi(\cdot)$ and $\psi(\cdot)$, the following sets are defined.

Definition 1. Define two function sets $\Phi=\{\phi(x) \mid \phi(x)$ is an odd function, $\phi(x) x>0, \phi(x)=0$ if and only if $x=0\}$, and $\Psi=\{\psi(x) \mid \psi(x)$ is a continuous function and increasing about $x, \psi(x)=\psi(y)$ if and only if $x=y\}$.

At the end of this section, two useful lemmas about $\phi(\cdot)$ and $\psi(\cdot)$ are introduced.

Lemma 2. Letting $\mathbf{a}=\left[\begin{array}{llll}a_{1} & a_{2} & \cdots & a_{n}\end{array}\right]^{T} \in \mathbb{R}^{n}, \mathbf{b}=$ $\left[\begin{array}{llll}b_{1} & b_{2} & \cdots & b_{n}\end{array}\right]^{T} \in \mathbb{R}^{n}$, and $C=\left[c_{i j}\right] \in \mathbb{R}^{n \times n}$, if matrix $C$ is symmetrical, that is, $c_{i j}=c_{j i}$, and odd function $\phi(x) \in \Phi$, then it is derived that

$$
\begin{array}{r}
\frac{1}{2} \sum_{i=1}^{n} \sum_{j=1}^{n} c_{i j}\left(a_{i}-a_{j}\right) \phi\left(b_{i}-b_{j}\right) \\
=\sum_{i=1}^{n} \sum_{j=1}^{n} c_{i j} a_{i} \phi\left(b_{i}-b_{j}\right) .
\end{array}
$$


Proof. Consider that

$$
\begin{gathered}
\frac{1}{2} \sum_{i=1}^{n} \sum_{j=1}^{n} c_{i j}\left(a_{i}-a_{j}\right) \phi\left(b_{i}-b_{j}\right) \\
=\frac{1}{2} \sum_{i=1}^{n} \sum_{j=1}^{n} c_{i j} a_{i} \phi\left(b_{i}-b_{j}\right) \\
-\frac{1}{2} \sum_{i=1}^{n} \sum_{j=1}^{n} c_{i j} a_{j} \phi\left(b_{i}-b_{j}\right) \\
=\frac{1}{2} \sum_{i=1}^{n} \sum_{j=1}^{n} c_{i j} a_{i} \phi\left(b_{i}-b_{j}\right) \\
+\frac{1}{2} \sum_{j=1}^{n} \sum_{i=1}^{n} c_{j i} a_{j} \phi\left(b_{j}-b_{i}\right) \\
=\sum_{i=1}^{n} \sum_{j=1}^{n} c_{i j} a_{i} \phi\left(b_{i}-b_{j}\right),
\end{gathered}
$$

where the facts that $c_{i j}=c_{j i}$ and $\phi(\cdot)$ is an odd function that is, $\phi\left(b_{j}-b_{i}\right)=-\phi\left(b_{i}-b_{j}\right)$, are used to obtain the equalities.

Using the same proof and the fact that $\psi\left(b_{j}\right)-\psi\left(b_{i}\right)=$ $-\left(\psi\left(b_{i}\right)-\psi\left(b_{j}\right)\right)$, the following lemma is obvious.

Lemma 3. Letting $\mathbf{a}=\left[\begin{array}{llll}a_{1} & a_{2} & \cdots & a_{n}\end{array}\right]^{T} \in \mathbb{R}^{n}, \mathbf{b}=$ $\left[\begin{array}{llll}b_{1} & b_{2} & \cdots & b_{n}\end{array}\right]^{T} \in \mathbb{R}^{n}$, and $C=\left[c_{i j}\right] \in \mathbb{R}^{n \times n}$, if matrix $C$ is symmetrical, function $\psi(x) \in \Psi$, then it follows that

$$
\begin{array}{r}
\frac{1}{2} \sum_{i=1}^{n} \sum_{j=1}^{n} c_{i j}\left(a_{i}-a_{j}\right)\left(\psi\left(b_{i}\right)-\psi\left(b_{j}\right)\right) \\
=\sum_{i=1}^{n} \sum_{j=1}^{n} c_{i j} a_{i}\left(\psi\left(b_{i}\right)-\psi\left(b_{j}\right)\right) .
\end{array}
$$

\section{Consensus Analysis}

In this section, the consensus and reference velocity consensus of second-order multiagent system (1) with the designed nonlinear protocol will be analyzed.

3.1. Consensus with Nonlinear Protocol. Second-order multiagent system (1) with protocol (4) is written as

$$
\begin{gathered}
\dot{x}_{i}(t)=v_{i}(t), \\
\dot{v}_{i}(t)=-\sum_{j=1}^{n} a_{i j}\left[\phi\left(x_{i}(t)-x_{j}(t)\right)\right. \\
\left.+\left(\psi\left(v_{i}(t)\right)-\psi\left(v_{j}(t)\right)\right)\right] .
\end{gathered}
$$

Theorem 4. For nonlinear protocol (4), suppose that functions $\phi(\cdot) \in \Phi$ and $\psi(\cdot) \in \Psi$. If information topology graph $\mathscr{G}$ is undirected and connected, then it is held that $x_{i}(t) \rightarrow x_{j}(t)$, $v_{i}(t) \rightarrow v_{j}(t)$, as $t \rightarrow \infty$; that is to say, multiagent system (8) achieves consensus asymptotically.
Proof. Choose the following Lyapunov function:

$$
V(t)=\frac{1}{2} \sum_{i=1}^{n} \sum_{j=1}^{n} a_{i j} \int_{0}^{x_{i}(t)-x_{j}(t)} \phi(s) d s+\frac{1}{2} \sum_{i=1}^{n} v_{i}^{2}(t) .
$$

The time derivative of the Lyapunov function (9) along any trajectory of (8) is

$\dot{V}(t)$

$$
\begin{aligned}
= & \frac{1}{2} \sum_{i=1}^{n} \sum_{j=1}^{n} a_{i j}\left(v_{i}(t)-v_{j}(t)\right) \phi\left(x_{i}(t)-x_{j}(t)\right) \\
& -\sum_{i=1}^{n} v_{i}(t)\left(\sum _ { j = 1 } ^ { n } a _ { i j } \left[\phi\left(x_{i}(t)-x_{j}(t)\right)\right.\right. \\
& \left.\left.+\left(\psi\left(v_{i}(t)\right)-\psi\left(v_{j}(t)\right)\right)\right]\right) \\
= & \sum_{i=1}^{n} \sum_{j=1}^{n} a_{i j} v_{i}(t) \phi\left(x_{i}(t)-x_{j}(t)\right) \\
& -\sum_{i=1}^{n} \sum_{j=1}^{n} a_{i j} v_{i}(t)\left[\phi\left(x_{i}(t)-x_{j}(t)\right)\right. \\
& \left.+\left(\psi\left(v_{i}(t)\right)-\psi\left(v_{j}(t)\right)\right)\right] \\
= & -\frac{1}{2} \sum_{i=1}^{n} \sum_{j=1}^{n} a_{i j}\left(v_{i}(t)-v_{j}(t)\right)\left(\psi\left(v_{i}(t)\right)-\psi\left(v_{j}(t)\right)\right),
\end{aligned}
$$

where the second equality and the last equality are derived from Lemmas 2 and 3 , respectively. Since $\left(v_{i}(t)-v_{j}(t)\right)\left(\psi\left(v_{i}(t)\right)-\psi\left(v_{j}(t)\right)\right) \geq 0$ and the information topology graph $\mathscr{G}$ is connected, it is derived that

$$
-\frac{1}{2} \sum_{i=1}^{n} \sum_{j=1}^{n} a_{i j}\left(v_{i}(t)-v_{j}(t)\right)\left(\psi\left(v_{i}(t)\right)-\psi\left(v_{j}(t)\right)\right) \leq 0 ;
$$

that is to say, $\dot{V}(t) \leq 0$. Letting $\dot{V}(t) \equiv 0$, it is easily seen that $v_{i}(t) \equiv v_{j}(t)$ and $\dot{v}_{i}(t) \equiv \dot{v}_{j}(t)$. Therefore, it follows that $\dot{v}(t) \in \operatorname{Span}(1 \times \varsigma)$, where $\varsigma$ is a constant and $v(t)=$ $\left[\begin{array}{llll}v_{1}(t) & v_{2}(t) & \cdots & v_{n}(t)\end{array}\right]^{\mathrm{T}}$. Furthermore, from $v_{i}(t) \equiv v_{j}(t)$, it is obtained that

$$
\begin{gathered}
\dot{v}_{i}(t) \equiv-\sum_{j=1}^{n} a_{i j}\left(\phi\left(x_{i}(t)-x_{j}(t)\right)\right), \\
(\mathbf{1} \times \varsigma)^{\mathrm{T}} \dot{v}_{i}(t) \equiv-\varsigma \sum_{i=1}^{n} \sum_{j=1}^{n} a_{i j}\left(\phi\left(x_{i}(t)-x_{j}(t)\right)\right) .
\end{gathered}
$$

Since information topology graph is undirected, that is, $a_{i j}=$ $a_{j i}$, and $\phi\left(x_{i}(t)-x_{j}(t)\right)=-\phi\left(x_{j}(t)-x_{i}(t)\right)$, it is held that

$$
\sum_{i=1}^{n} \sum_{j=1}^{n} a_{i j} \phi\left(x_{i}(t)-x_{j}(t)\right) \equiv 0,
$$


which implies that $(1 \times \varsigma)^{\mathrm{T}} \dot{v}(t) \equiv 0$. Thus, it means that $\dot{v}(t)$ is orthogonal to $\mathbf{1} \times \varsigma$. From the above discussion, it is concluded that $\dot{v}(t) \equiv 0$ which in turn implies that $-\sum_{j=1}^{n} a_{i j} \phi\left(x_{i}(t)-x_{j}(t)\right) \equiv 0$ from (12). Furthermore, it is obtained that $-\sum_{i=1}^{n} x_{i}(t) \sum_{j=1}^{n} a_{i j} \phi\left(x_{i}(t)-x_{j}(t)\right) \equiv 0$ which implies that

$$
-\frac{1}{2} \sum_{i=1}^{n} \sum_{j=1}^{n} a_{i j}\left(x_{i}(t)-x_{j}(t)\right) \phi\left(x_{i}(t)-x_{j}(t)\right) \equiv 0,
$$

from Lemma 2. For $\phi(x) x>0$ and information topology graph is connected, from (15), it is derived that

$$
a_{i j}\left(x_{i}(t)-x_{j}(t)\right) \phi\left(x_{i}(t)-x_{j}(t)\right) \equiv 0, \quad \forall i \neq j .
$$

As a result, it follows that $x_{i}(t) \equiv x_{j}(t)$, for all $i \neq j$. By LaSalle's invariance principle, it is concluded that $x_{i}(t) \rightarrow x_{j}(t)$, $v_{i}(t) \rightarrow v_{j}(t)$, for all $i \neq j$, as $t \rightarrow \infty$. The proof is ended.

When nonlinear protocol (4) is simplified as follows:

$$
u_{i}(t)=-\psi\left(v_{i}(t)\right)-\sum_{j=1}^{n} a_{i j} \phi\left(x_{i}(t)-x_{j}(t)\right),
$$

and the Lyapunov function (9) is chosen, the following conclusion is easily obtained.

Corollary 5. If the information topology graph $\mathscr{G}$ is undirected and connected, nonlinear functions $\phi(\cdot) \in \Phi$ and $\psi \in \Psi$, then the multiagent system (1) with protocol (17) can achieve consensus, namely, $x_{i}(t) \rightarrow x_{j}(t), v_{i}(t) \rightarrow v_{j}(t) \rightarrow 0$, asymptotically as $t \rightarrow \infty$.

In practice, since the position of each agent is easily measured relatively, nonlinear function $\phi(x)$ in protocol (4) can be assumed to be $\phi(x)=x$. Therefore, letting $\phi\left(x_{i}(t)-x_{j}(t)\right)=$ $x_{i}(t)-x_{j}(t)$, protocol (4) is reduced to

$$
u_{i}(t)=-\sum_{j=1}^{n} a_{i j}\left(x_{i}(t)-x_{j}(t)+\psi\left(v_{i}(t)\right)-\psi\left(v_{j}(t)\right)\right) .
$$

Applying protocol (18) to system (1), the following corollary is derived from Theorem 4 directly.

Corollary 6. For nonlinear protocol (18), suppose the function $\psi(\cdot) \in \Psi$. If the information topology graph $\mathscr{G}$ is undirected and connected, multiagent system (1) with protocol (18) achieves consensus asymptotically, namely, $x_{i}(t) \rightarrow x_{j}(t), v_{i}(t) \rightarrow$ $v_{j}(t)$, as $t \rightarrow \infty$.

Remark 7. The second-order multiagent system with linear protocol can be written as compact matrix form, and the consensus conditions can be derived by analysing the eigenvalues of the compact matrix [22]. When the secondorder multiagent system achieves consensus, the positions and velocities will, respectively, converge to a constant value which is relative to the initial position and velocity and the left eigenvector of the Laplacian matrix (see $[21,22])$.
However, the nonlinear protocol is different from the linear protocol. The second-order multiagent system with nonlinear protocol cannot be written as a compact matrix form. So, the proof of consensus is different from the method used in the linear protocol and is more difficult. When the consensus of the second-order multiagent system with nonlinear protocol is reached, the value which the positions and velocities converge to cannot be decided. The value is relative to the nonlinear function, the communication graph, and the initial position and velocity.

3.2. Consensus with Group Reference Velocity. In practice, given a reference velocity $v^{*}(t)$, the positions and velocities of the agents in multiagent system (1) are often required to achieve consensus and converge to $v^{*}(t)$; that is, $x_{i}(t) \rightarrow$ $x_{j}(t), v_{i}(t) \rightarrow v_{j}(t) \rightarrow v^{*}(t)$, for all $i \neq j$. Hence, the following nonlinear protocol is designed with a group reference velocity $v^{*}(t)$ as

$$
\begin{array}{r}
u_{i}(t)=\dot{v}^{*}(t)-\left(\psi\left(v_{i}(t)\right)-\psi\left(v^{*}(t)\right)\right) \\
-\sum_{j=1}^{n} a_{i j}\left(\phi\left(x_{i}(t)-x_{j}(t)\right)\right. \\
\left.+\psi\left(v_{i}(t)\right)-\psi\left(v_{j}(t)\right)\right) .
\end{array}
$$

Given a group reference velocity $v^{*}(t)$, second-order multiagent system (1) with protocol (19) is expressed as

$$
\begin{gathered}
\dot{x}_{i}(t)=v_{i}(t), \\
\dot{v}_{i}(t)=\dot{v}^{*}(t)-\left(\psi\left(v_{i}(t)\right)-\psi\left(v^{*}(t)\right)\right) \\
-\sum_{j=1}^{n} a_{i j}\left(\phi\left(x_{i}(t)-x_{j}(t)\right)\right. \\
\left.-\left(\psi\left(v_{i}(t)\right)-\psi\left(v_{j}(t)\right)\right)\right) .
\end{gathered}
$$

Theorem 8. For nonlinear protocol (19), consider functions $\phi(\cdot) \in \Phi$ and $\psi(\cdot) \in \Psi$. When information topology graph $\mathscr{G}$ is undirected and connected, multiagent system (20) achieves consensus; that is, $x_{i}(t) \rightarrow x_{j}(t), v_{i}(t) \rightarrow v_{j}(t) \rightarrow v^{*}(t)$, as $t \rightarrow \infty$.

Proof. Letting $\widetilde{v}_{i}(t)=v_{i}(t)-v^{*}(t), \widetilde{x}_{i}(t)=x_{i}(t)-\int_{0}^{t} v^{*}(s) d s$, system (20) is rewritten as

$$
\begin{gathered}
\dot{\tilde{x}}_{i}(t)=\widetilde{v}_{i}(t), \\
\dot{\tilde{v}}_{i}(t)=-\left(\psi\left(v_{i}(t)\right)-\psi\left(v^{*}(t)\right)\right) \\
-\sum_{j=1}^{n} a_{i j}\left(\phi\left(\tilde{x}_{i}(t)-\tilde{x}_{j}(t)\right)\right. \\
\left.-\left(\psi\left(v_{i}(t)\right)-\psi\left(v_{j}(t)\right)\right)\right) .
\end{gathered}
$$

Choose the following Lyapunov function:

$$
V(t)=\frac{1}{2} \sum_{i=1}^{n} \sum_{j=1}^{n} a_{i j} \int_{0}^{\tilde{x}_{i}(t)-\tilde{x}_{j}(t)} \phi(s) d s+\frac{1}{2} \sum_{i=1}^{n} \widetilde{v}_{i}^{2}(t) .
$$


Taking the derivative of the Lyapunov function (22) with respect to $t$ along the trajectory of (21) yields

$\dot{V}(t)$

$$
\begin{gathered}
=\frac{1}{2} \sum_{i=1}^{n} \sum_{j=1}^{n} a_{i j}\left(\widetilde{v}_{i}(t)-\widetilde{v}_{j}(t)\right) \phi\left(\tilde{x}_{i}(t)-\widetilde{x}_{j}(t)\right) \\
-\sum_{i=1}^{n} \widetilde{v}_{i}(t)\left(\begin{array}{c}
\left(\psi\left(v_{i}(t)\right)-\psi\left(v^{*}(t)\right)\right) \\
+\sum_{j=1}^{n} a_{i j}\left(\phi\left(\tilde{x}_{i}(t)-\tilde{x}_{j}(t)\right)\right. \\
\left.+\left(\psi\left(v_{i}(t)\right)-\psi\left(v_{j}(t)\right)\right)\right)
\end{array}\right.
\end{gathered}
$$

From Lemma 2, it is obtained that

$$
\begin{aligned}
\dot{V}(t)= & \frac{1}{2} \sum_{i=1}^{n} \sum_{j=1}^{n} a_{i j}\left(\widetilde{v}_{i}(t)-\widetilde{v}_{j}(t)\right) \phi\left(\tilde{x}_{i}(t)-\tilde{x}_{j}(t)\right) \\
& -\frac{1}{2} \sum_{i=1}^{n} \sum_{j=1}^{n} a_{i j}\left(\widetilde{v}_{i}(t)-\widetilde{v}_{j}(t)\right) \phi\left(\tilde{x}_{i}(t)-\widetilde{x}_{j}(t)\right) \\
& -\sum_{i=1}^{n} \widetilde{v}_{i}(t)\left(\psi\left(v_{i}(t)\right)-\psi\left(v^{*}(t)\right)\right) \\
& -\frac{1}{2} \sum_{i=1}^{n} \sum_{j=1}^{n} a_{i j}\left(\widetilde{v}_{i}(t)-\widetilde{v}_{j}(t)\right) \\
& \times\left(\psi\left(v_{i}(t)\right)-\psi\left(v_{j}(t)\right)\right) \\
= & -\sum_{i=1}^{n}\left(v_{i}(t)-v^{*}(t)\right)\left(\psi\left(v_{i}(t)\right)-\psi\left(v^{*}(t)\right)\right) \\
& -\frac{1}{2} \sum_{i=1}^{n} \sum_{j=1}^{n} a_{i j}\left(v_{i}(t)-v_{j}(t)\right) \\
& \times\left(\psi\left(v_{i}(t)\right)-\psi\left(v_{j}(t)\right)\right) .
\end{aligned}
$$

Since function $\psi(\cdot) \in \Psi$ and the information topology graph $\mathscr{G}$ is undirected and connected, there exist the following facts that

$$
\begin{gathered}
-\sum_{i=1}^{n}\left(v_{i}(t)-v^{*}(t)\right)\left(\psi\left(v_{i}(t)\right)-\psi\left(v^{*}(t)\right)\right) \leq 0, \\
-\frac{1}{2} \sum_{i=1}^{n} \sum_{j=1}^{n} a_{i j}\left(v_{i}(t)-v_{j}(t)\right)\left(\psi\left(v_{i}(t)\right)-\psi\left(v_{j}(t)\right)\right) \leq 0 .
\end{gathered}
$$

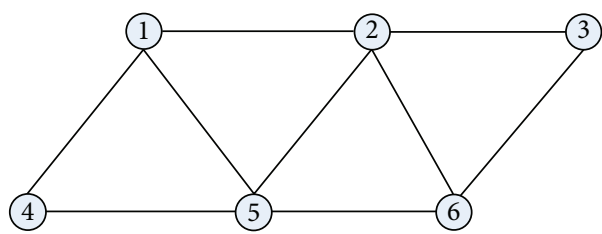

FIGURE 1: Communication topology graph.

Combing (25), it follows that $\dot{V}(t) \leq 0$. When $\dot{V}(t) \equiv 0$, it is clear that $v_{i}(t) \equiv v_{j}(t), v_{i}(t) \equiv v^{*}(t)$. Then, it is derived that

$$
\begin{gathered}
-\sum_{j=1}^{n} \phi\left(\tilde{x}_{i}(t)-\tilde{x}_{j}(t)\right) \equiv 0, \\
-\sum_{i=1}^{n} \tilde{x}_{i}(\mathrm{t}) \sum_{j=1}^{n} a_{i j} \phi\left(\tilde{x}_{i}(t)-\tilde{x}_{j}(t)\right) \equiv 0 .
\end{gathered}
$$

Note that (27) implies that

$$
-\frac{1}{2} \sum_{i=1}^{n} \sum_{j=1}^{n} a_{i j}\left(\tilde{x}_{i}(t)-\tilde{x}_{j}(t)\right) \phi\left(\tilde{x}_{i}(t)-\tilde{x}_{j}(t)\right) \equiv 0,
$$

from Lemma 2. Due to the connected information graph $\mathscr{G}$, it is derived from (28) that

$$
\left(\tilde{x}_{i}(t)-\tilde{x}_{j}(t)\right) \phi\left(\tilde{x}_{i}(t)-\tilde{x}_{j}(t)\right) \equiv 0 .
$$

As a result, it is concluded that $\tilde{x}_{i}(t) \equiv \tilde{x}_{j}(t)$ which is equivalent to $x_{i}(t) \equiv x_{j}(t)$. Making use of LaSalle's invariance principle, it is concluded that $x_{i}(t) \rightarrow x_{j}(t), v_{i}(t) \rightarrow$ $v_{j}(t) \rightarrow v^{*}(t)$, as $t \rightarrow \infty$. The proof is completed.

\section{Numerical Examples}

In this section, a number of simulations are given to illustrate the effectiveness of the results proposed in the paper.

Example 9. Consider the communication topology graph $\mathscr{G}$ as described in Figure 1. Graph $\mathscr{G}$ has eight nodes, and the edges denote the information interchange between agents. For simplicity, it is assumed that graph $\mathscr{G}$ has $0-1$ weights. To simulate numerical examples, choose

$$
\phi(x)= \begin{cases}x^{2} & \text { if } x>1 \\ \sqrt{x} & \text { if } 0<x \leq 1 \\ -\sqrt{-x} & \text { if }-1<x \leq 0 \\ -x^{2} & \text { if } x \leq-1\end{cases}
$$

and $\psi(x)=x^{3}$ in the nonlinear protocol (4), and it is clear that $\phi \in \Phi$ and $\psi \in \Psi$. Therefore, the nonlinear protocol (4) can be achieved asymptotically for multiagent system (1) from Theorem 4. Figure 2 shows that the velocity and position trajectories of second-order multiagent system (8) converge to consensus, respectively. In addition, Figure 3 describes that both the velocity and position trajectories of second-order multiagent system (1) with protocol (18) achieve consensus. 

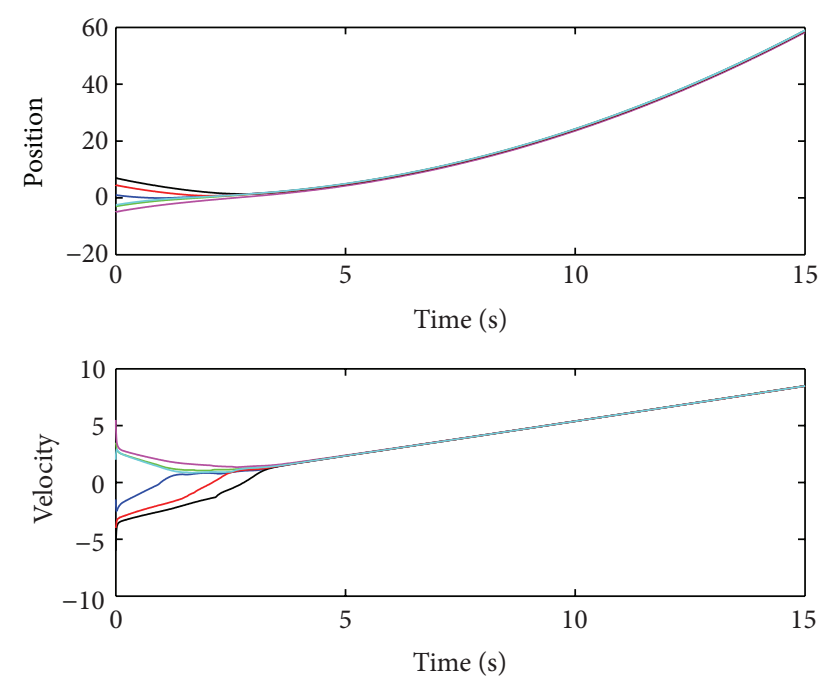

FIGURE 2: State trajectories of multiagent system (8).
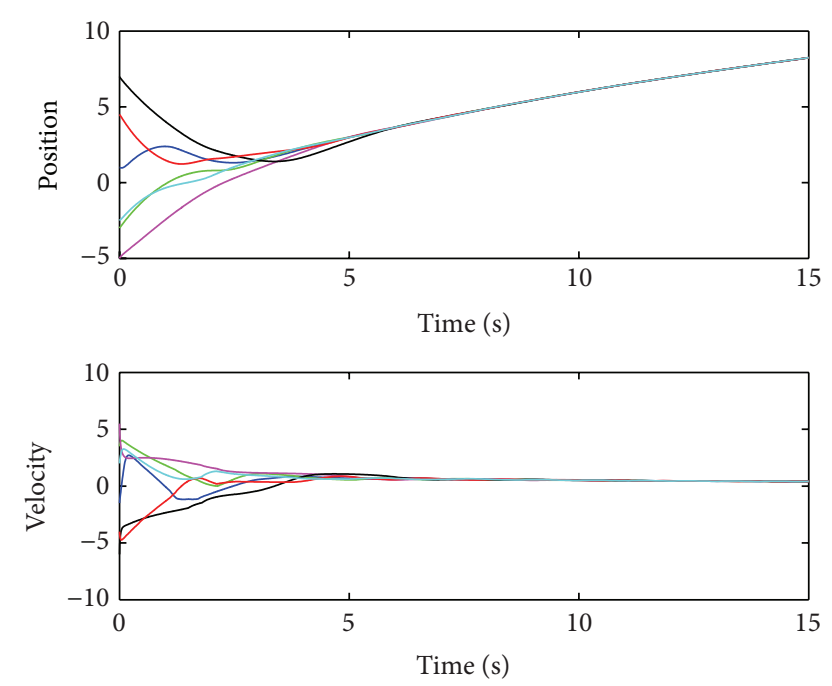

FIGURE 3: State trajectories of multiagent system (1) with protocol (18).

Choose a reference velocity $v^{*}(t)=\sin (3 * t)+\cos (t)$, and using protocol (19), multiagent system (1) can achieve consensus asymptotically. Figure 4 describes the trajectories of velocity and position of multiagent system (1) with protocol (19), respectively. It is seen that the trajectories of velocity follow the reference velocity $v^{*}(t)$ successfully.

\section{Conclusions}

This paper provided consensus analysis for the second-order multiagent system with nonlinear protocol. As an extension of linear protocols, a nonlinear protocol was designed to achieve consensus. In addition, the protocol was also used to achieve reference velocity consensus for second-order multiagent system. The analysis mainly relied on some tools from algebraic graph and control theory. Through using the
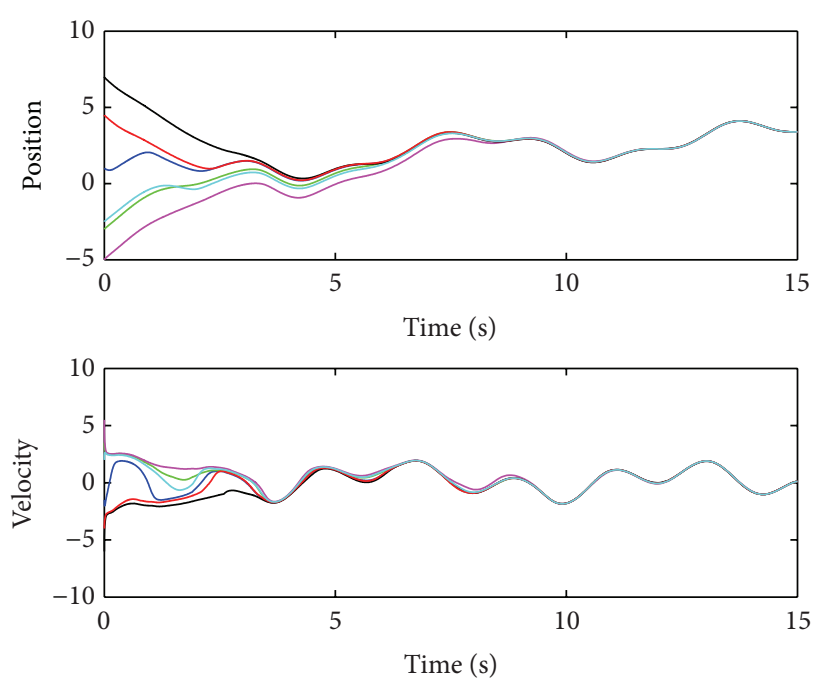

FIGURE 4: State trajectories of multiagent system (20).

Lyapunov theory and LaSalle's invariance principle, sufficient conditions for consensus are derived. Simulation examples show that when the nonlinear function satisfies the assumption, the second-order multiagent can achieve consensus and reference velocity consensus.

\section{Acknowledgment}

This work was supported by the Research Funds of Ningxia University Introducing Talent (no. BQD2012004).

\section{References}

[1] W. Lu, F. Atay, and J. Jost, "Synchronization of discretetime dynamical networks with time-varying couplings," SIAM Journal on Mathematical Analysis, vol. 39, no. 2, pp. 1231-1259, 2007.

[2] S. Barbarossa and G. Scutari, "Decentralized maximumlikelihood estimation for sensor networks composed of nonlinearly coupled dynamical systems," IEEE Transactions on Signal Processing, vol. 55, no. 7, part 1, pp. 3456-3470, 2007.

[3] T. Chen and Z. Zhu, "Exponential synchronization of nonlinear coupled dynamical networks," International Journal of Bifurcation and Chaos in Applied Sciences and Engineering, vol. 17, no. 3, pp. 999-1005, 2007.

[4] W. Lu and T. Chen, "Synchronization of coupled connected neural networks with delays," IEEE Transactions on Circuits and Systems. I., vol. 51, no. 12, pp. 2491-2503, 2004.

[5] X. Liu and T. Chen, "Exponential synchronization of nonlinear coupled dynamical networks with a delayed coupling," Physica A, vol. 381, no. 15, pp. 82-92, 2007.

[6] W. Yu, G. Chen, J. Lü, and J. Kurths, "Synchronization via pinning control on general complex networks," SIAM Journal on Control and Optimization, vol. 51, no. 2, pp. 1395-1416, 2013.

[7] D. Bauso, L. Giarré, and R. Pesenti, "Non-linear protocols for optimal distributed consensus in networks of dynamic agents," Systems \& Control Letters, vol. 55, no. 11, pp. 918-928, 2006.

[8] F. Xiao, L. Wang, and Y. Jia, "Fast information sharing in networks of autonomous agents," in Proceedings of the American 
Control Conference (ACC '08), pp. 4388-4393, Seattle, Wash, USA, June 2008.

[9] R. Olfati-Saber and R. M. Murray, "Consensus problems in networks of agents with switching topology and time-delays," IEEE Transactions on Automatic Control, vol. 49, no. 9, pp. 15201533, 2004.

[10] X. Liu, T. Chen, and W. Lu, "Consensus problem in directed networks of multi-agents via nonlinear protocols," Physics Letters A, vol. 373, no. 35, pp. 3122-3127, 2009.

[11] Q. Hui and W. M. Haddad, "Distributed nonlinear control algorithms for network consensus," Automatica, vol. 44, no. 9, pp. 2375-2381, 2008.

[12] Y. Shang, "On consensus of nonlinear multi-agent systems under directed interaction topologies," in Proceedings of the International Conference on Cyber-Enabled Distributed Computing and Knowledge Discovery (CyberC '09), pp. 128-131, Beijing, China, October 2009.

[13] M. Li and X. Guan, "Nonlinear consensus protocols for multiagent systems based on centre manifold reduction," Chinese Physics B, vol. 18, no. 8, pp. 3355-3366, 2009.

[14] G. Shi and Y. Hong, "Global target aggregation and state agreement of nonlinear multi-agent systems with switching topologies," Automatica, vol. 45, no. 5, pp. 1165-1175, 2009.

[15] R. Sepulchre, "Consensus on nonlinear spaces," Annual Reviews in Control, vol. 35, no. 1, pp. 56-64, 2011.

[16] W. Yu, G. Chen, and M. Cao, "Consensus in directed networks of agents with nonlinear dynamics," IEEE Transactions on Automatic Control, vol. 56, no. 6, pp. 1436-1441, 2011.

[17] J. Zhou and Q. Wang, "Distributed discrete-time nonlinear consensus protocols," in Proceedings of the 48th IEEE Conference on Decision and Control and 28th Chinese Control Conference (CDC/CCC '09), pp. 4759-4764, Shanghai, China, December 2009.

[18] W. Yu, G. Chen, M. Cao, and J. Kurths, "Second-Order consensus for multiagent systems with directed topologies and nonlinear dynamics," IEEE Transactions on Systems, Man, and Cybernetics B, vol. 40, no. 3, pp. 881-891, 2010.

[19] Q. Song, J. Cao, and W. Yu, "Second-order leader-following consensus of nonlinear multi-agent systems via pinning control," Systems \& Control Letters, vol. 59, no. 9, pp. 553-562, 2010.

[20] W. Ren, "On consensus algorithms for double-integrator dynamics," IEEE Transactions on Automatic Control, vol. 53, no. 6, pp. 1503-1509, 2008.

[21] W. Ren and E. Atkins, "Distributed multi-vehicle coordinated control via local information exchange," International Journal of Robust and Nonlinear Control, vol. 17, no. 10-11, pp. 1002-1033, 2007.

[22] W. Yu, G. Chen, and M. Cao, "Some necessary and sufficient conditions for second-order consensus in multi-agent dynamical systems," Automatica, vol. 46, no. 6, pp. 1089-1095, 2010. 


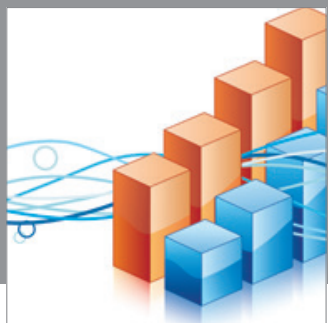

Advances in

Operations Research

mansans

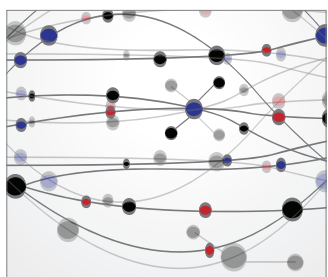

The Scientific World Journal
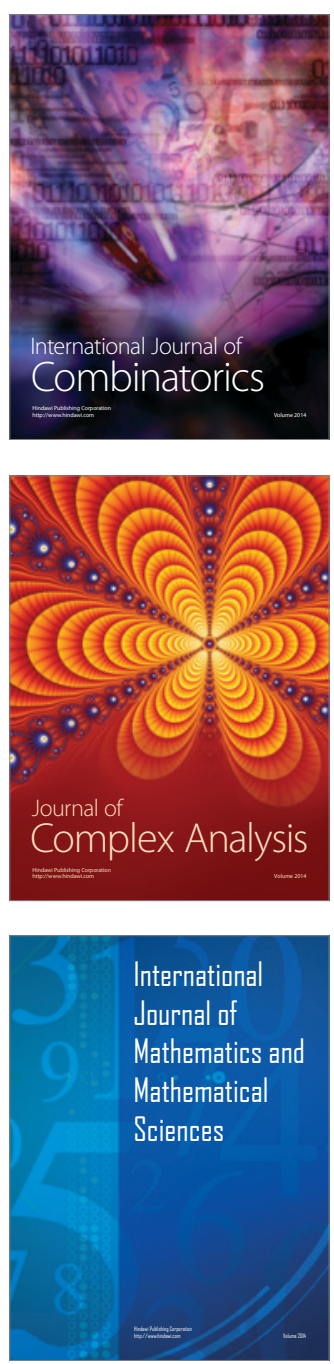
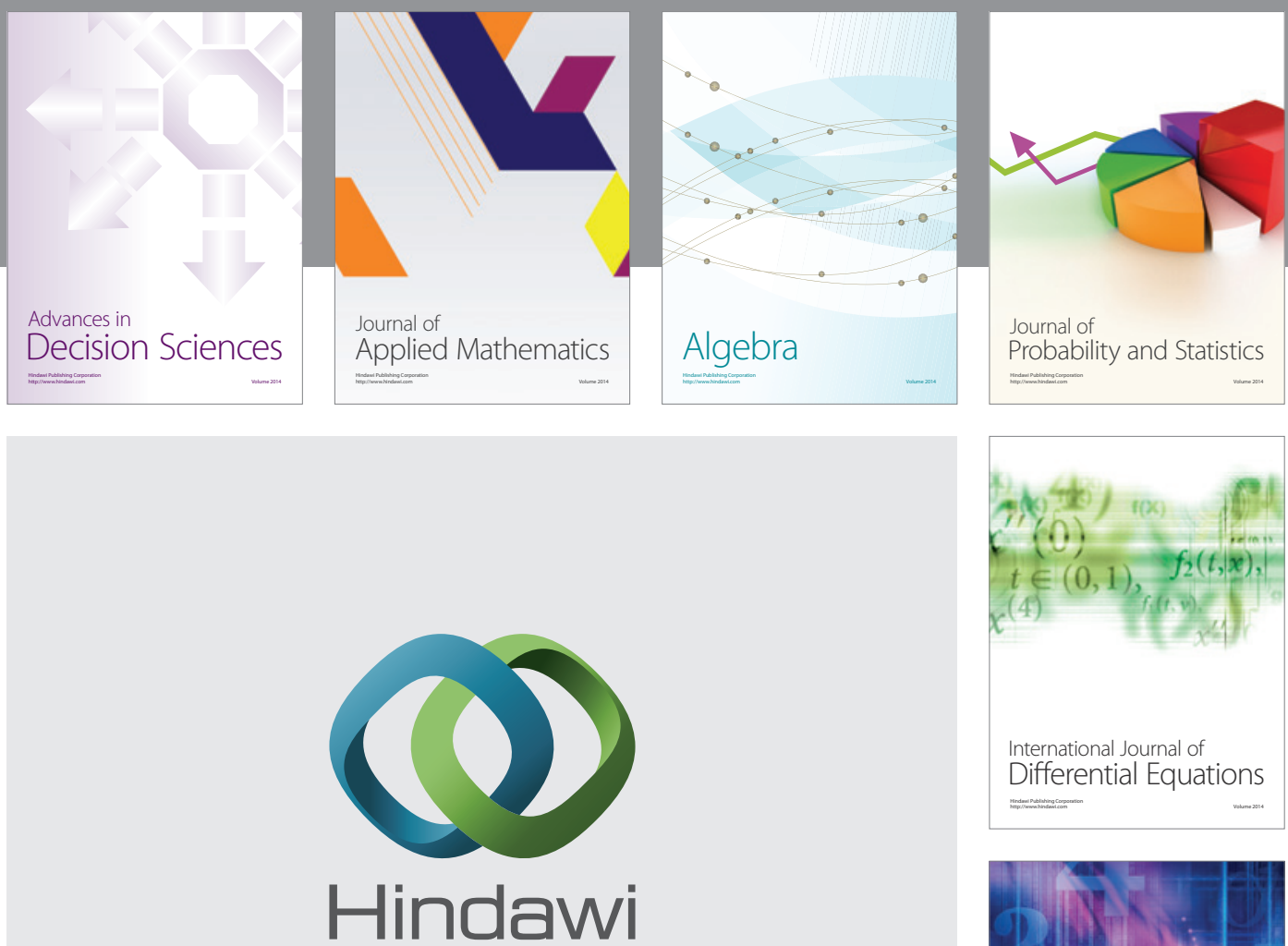

Submit your manuscripts at http://www.hindawi.com
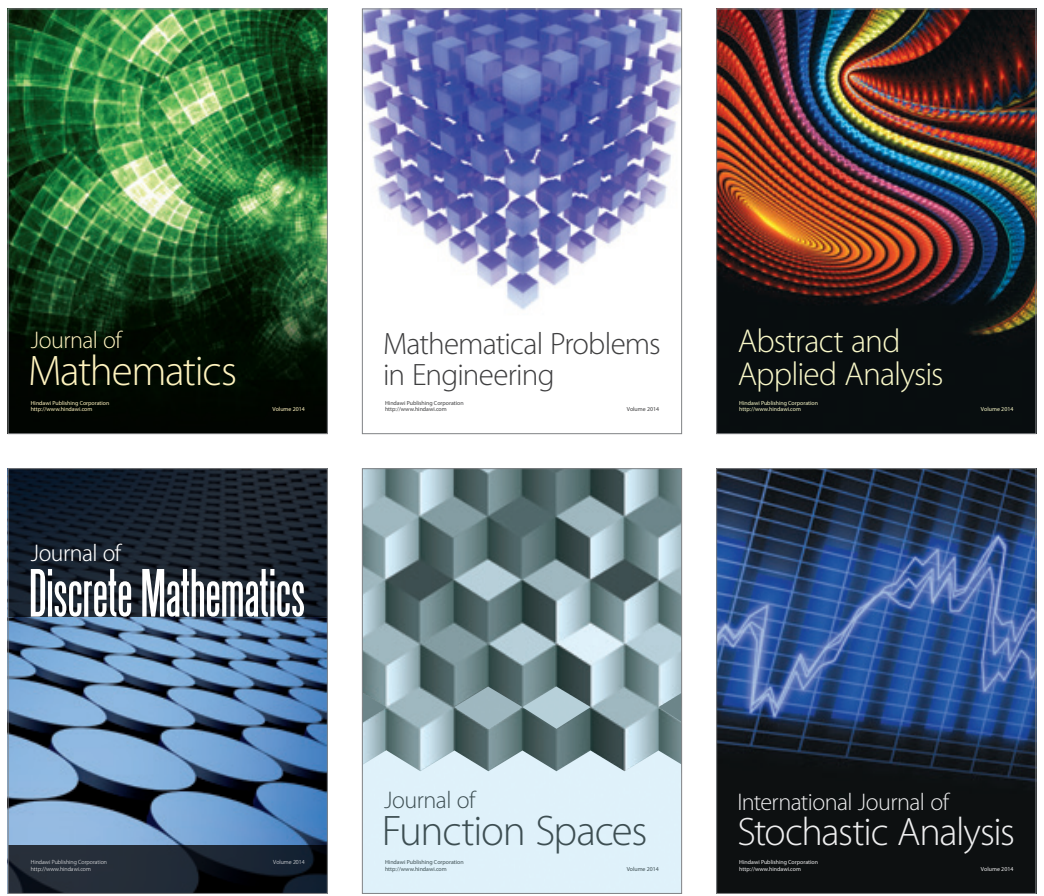

Journal of

Function Spaces

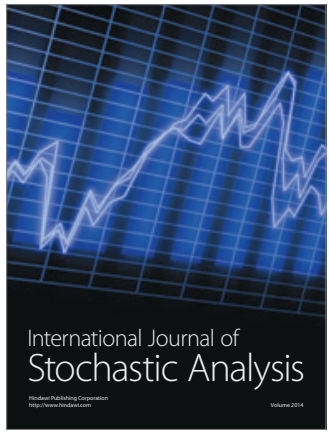

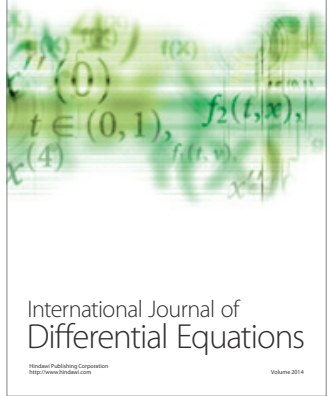
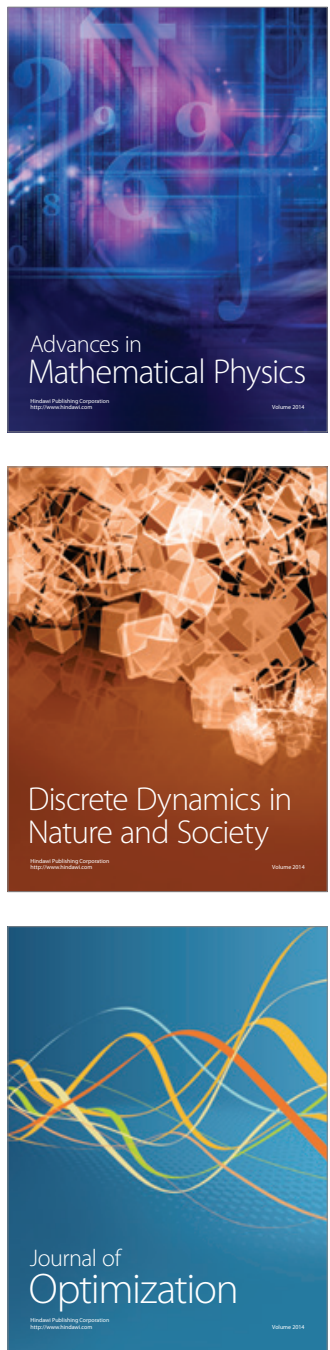